\title{
Effects of Dobutamine and Levosimendan on Systolic Time Intervals in Patients with Decompensated Heart Failure
}

\author{
Aydin Nadir (Corresponding author) \\ Bezmialem Vakif University, Faculty of Medicine, \\ Department of Cardiology, Maltepe/Istanbul, Turkey \\ ORCID ID: 0000-0003-2486-4009, E-mail: aydin_nadir@yahoo.com \\ Kadir Ugur Mert \\ Eskişehir Osmangazi University, Faculty of Medicine, \\ Department of Cardiology, Eskisehir, Turkey \\ ORCID ID: 0000-0002-1331-5365, E-mail: kugurmert@gmail.com \\ Bektas Morrad \\ Eskişehir Osmangazi University, Faculty of Medicine, \\ Department of Cardiology, Eskisehir, Turkey \\ ORCID ID: 0000-0002-6564-7185,E-mail: dr.bektash@hotmail.com \\ Fezan Mutlu \\ Eskişehir Osmangazi University, Faculty of Medicine, \\ Department of Biostatistic, Eskisehir, Turkey \\ ORCID ID: 0000-0002-9339-4031,E-mail: fezan.mutlu@ hotmail.com \\ Yuksel Cavusoglu \\ Eskişehir Osmangazi University, Faculty of Medicine, \\ Department of Cardiology, Eskisehir, Turkey \\ ORCID ID: 0000-0002-4027-9873, E-mail: yukselc@ogu.edu.tr
}

\begin{abstract}
Background/Objective: Levosimendan represents an alternative to other positive inotropic agents based on its different mechanisms of action and favorable electrophysiological properties. This study compared the effects of levosimendan and dobutamine on echocardiographic parameters in heart failure patients with acute decompensation necessitating positive inotropic support.

Methods: Patients with acute decompensated heart failure were randomized to receive inotropic support with either levosimendan $(n=25)$ or dobutamine $(n=25)$. Treatment groups were compared in terms of echocardiography measurements including tissue Doppler examination findings, systolic time interval assessments and diastolic parameters. In addition, groups were compared for demographic features, clinical characteristics and laboratory findings.

Results: Among tissue Doppler measurements, Sm-lateral and Sm-septal significantly increased after treatment in both groups. E/E' lateral and E/E' septal significantly decreased only in the levosimendan group. Among systolic time interval parameters, increasing in left ventricular ejection time and shortening in pre-ejection period are similar in both groups, however a significant decrease in QS2i was observed in the levosimendan group. Levosimendan treatment was associated with significant decreases in blood pressures along with a significant increase in ejection fraction. Dobutamine treatment on the other hand resulted in significant increases in blood pressure, heart rate and ejection fraction.

Conclusions: Our findings suggest that levosimendan and dopamine treatments are associated with only marginal differences in echocardiographic parameters. This study suggests that both levosimendan and dobutamine are almost equally effective in systolic time intervals. However, levosimendan appears to have additional advantage over dobutamine in shortening QS2i, indicating a fairly strong positive inotropic effect.
\end{abstract}

Keywords: decompensated heart failure, dobutamine, levosimendan, inotropic support, echocardiography, tissue doppler, systolic time intervals

DOI: $10.7176 /$ JSTR/5-3-07

52 | P a g e

www.iiste.org 


\title{
INTRODUCTION
}

Heart failure is a syndrome associated with recurrent hospitalizations and poor prognosis ${ }^{1,2}$. It has a prevalence rate of 0.4 to $2 \%$ and $>10 \%$ in subjects under 65 years of age and in those $\geq 65$ years of age, respectively ${ }^{3}$.

Most hospitalizations in patients with chronic heart failure are result of acute decompensation. Acute exacerbations of chronic heart failure due to left ventricular dysfunction are frequently treated with diuretics, intravenous vasodilators, and positive inotropic agents. The latter class of medications is administered in the presence of hypoperfusion and congestion signs and in patients with low systolic blood pressure (SBP) or measured cardiac index 4 .

In recent years, a novel class of drugs termed "calcium sensitizers" with favorable hemodynamic characteristics has been introduced for use. Among these, levosimendan has been the most widely utilized agent clinically ${ }^{5}$. It is a novel inotropic drug with myocardial calcium sensitizing and vasodilator effects developed for the short-term treatment of these patients. It has been proposed to mitigate the untoward effects of other positive inotropic agents, as it is not associated with increased intracellular calcium concentrations ${ }^{6}$. Furthermore, it has been suggested to provide a viable alternative to other positive inotropic agents based on its different mechanisms of action and favorable electrophysiological properties 5 . Dobutamine is a positive inotropic agent that is widely utilized for the treatment of decompensated heart failure to provide symptomatic benefits, although it is known to be associated with induction of arrhythmias and elevated mortality risk. Randomized clinical studies of have confirmed that levosimendan improves symptoms as well as systolic and diastolic functions, and lowers mortality as compared to placebo and dobutamine ${ }^{7}$. However, until now no large-scale, randomized, double-blind studies have been undertaken to compare levosimendan and dobutamine with regard to their effects on systolic interval.

This study was carried out to comparatively assess the effects of levosimendan and dobutamine on echocardiographic parameters including systolic interval in decompensated heart failure patients hospitalized with clinical signs and symptoms necessitating the use of positive inotropic support.

\section{METHODS}

\section{Patients}

Patients with acute decompensated heart failure with NYHA III-IV functional capacity and LVEF $<\% 35$ who received inotropic support with either levosimendan or dobutamine due to unsatisfactory response to optimal oxygen, diuretic (intravenous furosemide) and vasodilator (intravenous nitrate) treatment were included. Exclusion criteria were as follows: acute coronary syndrome, advanced valvular disease, hypertrophic obstructive or restrictive cardiomyopathy, severe hepatic or renal failure, atrial fibrillation or flatter and patients with pacemaker. The study protocol was approved by the local ethics committee and all patients gave informed consent prior to study entry.

\section{Study medications}

Patients were randomized to receive either levosimendan (Simdax ${ }^{\circledR}$, Orion Pharma, Finland) (n=25) or dobutamine (Dobutamin ${ }^{\circledR}$, Abott, IL, USA) $(n=25)$ infusion for inotropic support with 1:1 ratio. A 12 $\mathrm{mcg} / \mathrm{kg}$ loading dose of levosimendan was administered in 10 minutes, which was followed by 0.1 $\mathrm{mcg} / \mathrm{kg} / \mathrm{min}$ infusion for one hour. If tolerated, the dose was increased to $0.2 \mathrm{mcg} / \mathrm{kg} / \mathrm{min}$ and administered for an additional 23 hours. If not tolerated, the dose was decreased to $0.05 \mathrm{mcg} / \mathrm{kg} / \mathrm{min}$ and then titrated according to systolic blood pressure aiming to reach $0.2 \mathrm{mcg} / \mathrm{kg} / \mathrm{min}$ dose. Dobutamine was administered at a dose of $10 \mathrm{mcg} / \mathrm{kg} / \mathrm{min}$ for 24 hours, without any preceding loading dose.

\begin{abstract}
Assessments
Demographical and clinical data were recorded at baseline. Biochemical and hematological laboratory data, blood pressure and heart rate measurements were recorded at baseline and after treatment. All patients had echocardiography examinations before and 24 hours after infusions. In addition, functional capacity was assessed using 6-minute walk test before and after treatment by recording the distance covered during 6 minutes of walking in meters.
\end{abstract}

\section{Echocardiography examinations}

Tissue Doppler examination

Pulse wave (PW) Doppler recordings were taken at the left ventricular basal-septal and basal-lateral walls using tissue Doppler mode in four-chamber view using two-dimensional transthoracic echocardiography. The following parameters were analyzed during tissue Doppler examination: systolic wave velocity

53 | P a g e www.iiste.org 
$(\mathrm{Sm})$, early diastolic (Em, E') and late diastolic (Am, A') wave velocity, and S time (duration of time from the beginning to the end of the $\mathrm{S}$ wave). Again, using two-dimensional transthoracic echocardiography in apical four-chamber view, the early filling velocity ( $E$ wave) was inferred from the mitral inflow pattern, and the septal and lateral E/E' (Em) ratios of basal lateral and basal septal early diastolic waves were determined with tissue Doppler.

Systolic time interval assessments

Patients were monitored using electrocardiogram (ECG). M-mode recordings at the parasternal long axis were made with two-dimensional transthoracic echocardiography at the level of aortic valve. Using the EKG q wave, measurements for the pre-ejection period (PEP, period before aortic valve opening), left ventricular ejection time (LVET, time between the opening and closure of the aortic valve), and QS2 $(\mathrm{QS} 2=\mathrm{PEP}+\mathrm{LVET}$, the time from the Q wave of ECG to the closure of the aortic valve) were performed. Additionally, PEP and QS2 values corrected for the heart rate were recorded as CPEP and cQS2, respectively.

\section{Diastolic parameters}

Using pulse wave (PW) Doppler at five parasternal windows, the early filling velocity (E wave) and atrial contraction velocity (A wave) were measured and E/A ratios were estimated based on mitral inflow pattern; also measured were the DT (mitral deceleration time) from mitral early filling peak velocity to the end of early filling velocity, IVCT (isovolumetric contraction time) from the end of the velocity of atrial contraction (A) to the beginning of aortic valve filling velocity, ejection time (ET) from the beginning of the aortic valve flow to the end of the aortic valve flow, and IVRT (isovolumetric relaxation time) from the end of aortic valve flow velocity to the beginning of the early filling velocity based on the mitral inflow pattern. Finally, the Tei index was estimated based on these measurements as follows: Tei index $=($ ICT-IRT $) /$ ET.

\section{Statistical analysis}

Statistical Package for Social Sciences (SPSS) for Windows version 16.0 was used for statistical analyses. Data were presented in mean \pm standard deviation or number (percent), where appropriate. Categorical variables were compared using chi-square test. Inter-group differences of continuous variables were compared with student-t test or Mann-Whitney U test, depending on the normality of distribution. Intra-group differences between before and after treatment were tested using paired $t$ test. A $p$ value $<0.05$ was considered an indication of statistical significance.

\section{RESULTS}

Table 1 shows demographical and clinical characteristics of the patients at baseline. The two treatment groups did not differ regarding demographic characteristics, co-morbidities, family history, smoking habit, ejection fraction, body mass index, and type of cardiomyopathy ( $p>0.05$ for all). In addition, groups did not differ in terms of hematological and biochemical findings at baseline, including $\mathrm{Na}$, $\mathrm{K}$, blood urinary nitrogen, creatinine, Hs-CRP, Prp-BNP, Troponin, CK-MB, myoglobulin, hemoglobulin, hematocrit, and proteinuria levels and leukocyte and platelet counts ( $p>0.05$ for all).

\section{Changes in clinical and laboratory parameters}

Table 2 shows the changes in clinical parameters and comparison of the two treatment groups. There was significant improvement in 6-minute walk test in both groups when compared to baseline; however, groups did not differ regarding improvement in walking test results. Levosimendan treatment was associated with significant decreases in both systolic and diastolic pressures along with a significant increase in ejection fraction. Dobutamine treatment on the other hand resulted in significant increases in systolic blood pressure, heart rate and ejection fraction. In the dobutamine group, proteinuria decreased significantly after treatment $(332,14 \pm 223$ vs. $249,54 \pm 162 \mathrm{~g} / \mathrm{d}, \mathrm{p}=0.004)$. And also BUN $(24,7 \pm 8,29$ vs. $29,23 \pm 10,1 \mathrm{mg} / \mathrm{dl}, \mathrm{p}=0,031)$ and creatinine $(0,98 \pm 0,26 \mathrm{vs} .1 .16 \pm 0,27 \mathrm{mg} / \mathrm{dl}, \mathrm{p}=0,016)$ levels increased significantly with treatment. No other significant changes were observed in biochemical and hematological parameters ( $p>0.05$ for all). 
Table 1. Demographical and clinical characteristics of the patients

\begin{tabular}{lcccc} 
& $\begin{array}{c}\text { All patients } \\
(\mathbf{n = 5 0})\end{array}$ & $\begin{array}{c}\text { Levosimendan } \\
(\mathbf{n = 2 5})\end{array}$ & $\begin{array}{c}\text { Dobutamine } \\
(\mathbf{n = 2 5})\end{array}$ & p \\
\cline { 2 - 5 } Age, y (mean $\pm \mathrm{SD})$ & $61,7 \pm 10,3$ & $63 \pm 10,3$ & $59,8 \pm 10,4$ & $\mathrm{NS}$ \\
Male gender & $42(\% 84)$ & $21(\% 84)$ & $21(\% 84)$ & $\mathrm{NS}$ \\
Obesity & $18(\% 36)$ & $7(\% 28)$ & $11(\% 44)$ & $\mathrm{NS}$ \\
Hypertension & $41(\% 82)$ & $18(\% 72)$ & $23(\% 92)$ & $\mathrm{NS}$ \\
Hyperlipidemia & $39(\% 78)$ & $19(\% 76)$ & $20(\% 80)$ & $\mathrm{NS}$ \\
Diabetes & $23(\% 46)$ & $9(\% 36)$ & $14(\% 56)$ & $\mathrm{NS}$ \\
Smoking & $22(\% 44)$ & $13(\% 52)$ & $9(\% 36)$ & $\mathrm{NS}$ \\
Family history & $6(\% 12)$ & $4(\% 16)$ & $2(\% 8)$ & $\mathrm{NS}$ \\
Body mass index, kg/m ${ }^{2}(\operatorname{mean} \pm \mathrm{SD})$ & $28,3 \pm 3,6$ & $27,43 \pm 3,65$ & $29,19 \pm 3,43$ & $\mathrm{NS}$ \\
Ejection fraction, \% (mean $\pm \mathrm{SD})$ & $26,9 \pm 5.2$ & $27,9 \pm 4,68$ & $26 \pm 5,59$ & $\mathrm{NS}$ \\
Ischemic cardiomyopathy & $43(\% 86)$ & $22(\% 88)$ & $21(\% 84)$ & $\mathrm{NS}$ \\
Dilated cardiomyopathy & $7(\% 14)$ & $3(\% 12)$ & $4(\% 16)$ & $\mathrm{NS}$ \\
\hline
\end{tabular}

Unless otherwise stated, data presented in n (\%), NS: non-significant

Table 2. Changes in clinical parameters after treatment

\begin{tabular}{lccccccc} 
& \multicolumn{3}{c}{ Dobutamine (n=25) } & \multicolumn{3}{c}{ Levosimendan (n=25) } & p** \\
\cline { 2 - 7 } & Pre- & Post- & p* & Pre- & Post- & p* & \\
\cline { 2 - 7 } 6-minute walk test, m & $179,08 \pm 68,79$ & $222,4 \pm 79,64$ & 0,000 & $191,16 \pm 83$ & $232,72 \pm 88$ & 0,000 & 0,835 \\
SBP (mmHg) & $106,1 \pm 13,01$ & $114,4 \pm 13,75$ & 0,001 & $122,6 \pm 14,22$ & $100,9 \pm 12,7$ & 0,000 & 0,000 \\
DBP (mmHg) & $67,92 \pm 10,19$ & $70,88 \pm 9,7$ & 0,06 & $79,68 \pm 14,27$ & $69,8 \pm 9,42$ & 0,002 & 0,000 \\
Heart rate (beat/min) & $81,36 \pm 14,75$ & $89,64 \pm 15,08$ & 0,000 & $84,92 \pm 11,91$ & $82,28 \pm 10,4$ & 0,192 & 0,000 \\
Ejection fraction. \% & $27,96 \pm 4,68$ & $30,8 \pm 4,72$ & 0,000 & $26,0 \pm 5,59$ & $30,64 \pm 5,49$ & 0,000 & 0,032 \\
\hline
\end{tabular}

*pre- versus post-treatment. **dobutamine versus levosimendan group SBP. systolic blood pressure; DBP. diastolic blood pressure

\section{Changes in echocardiography parameters}

Table 3 shows changes in echocardiography parameters after treatment and compares the two groups. Among tissue Doppler measurements, Sm lat and Sm sept significantly increased after treatment in both groups, however the groups did not differ in terms of changes in these two parameters. E/E' lat and E/E' sept significantly decreased only in the levosimendan group, but did not change in the dobutamine group. None of the other tissue doppler parameters 
significantly changed after treatment in either of the groups. Similarly, none of the diastolic echocardiographic parameters significantly changed in either of the groups after treatment.

Among systolic time interval parameters, a significant change in PEP, LVET, PEP/LVET and cPEP was revealed in both groups, however the groups did not differ in terms of changes in these parameters. Besides, a significant decrease in QS2i was observed only in the levosimendan group, and no other changes in systolic time interval parameters could reach statistical significance.

Table 3. Changes in echocardiography parameters

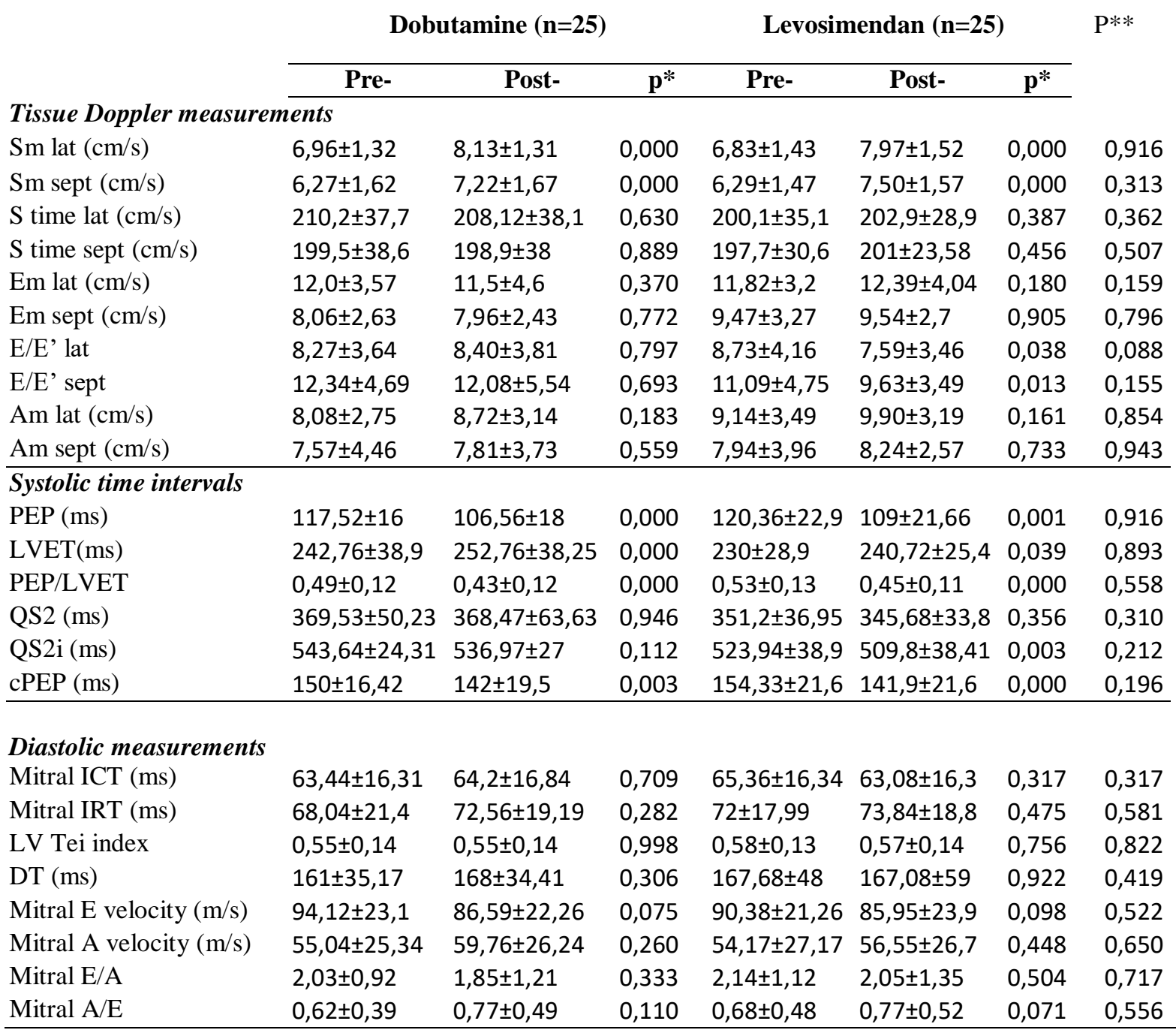

*pre- versus post-treatment. $* *$ dobutamine versus levosimendan group

Sm lat, mitral peak systolic velocity lateral wall; Sm sept, mitral peak systolic velocity septal wall; Em lat, mitral early diastolic velocity lateral wall; Em sept, mitral early diastolic velocity septal wall; Am lat, mitral late diastolic velocity lateral wall; Am sept, mitral late diastolic velocity septal wall; E/E' lat, mitral E/E' ratio lateral wall; E/E' sept, mitral E/E' ratio septal wall; PEP, pre-ejection period; LVET, left ventricular ejection time; PEP/VET, PEP/VET ratio; QS2, electromechanical systole; QS2i, corrected electromechanical systole; cPEP, corrected pre-ejection period; cLVET, corrected left ventricular ejection time; ICT, isovolumetric contraction time; IRT, isovolumetric relaxation time; LV Tei index, left ventricular Tei index; $\mathrm{DT}$, deceleration time; Mitral E/A, mitral E/A' ratio; Mitral A/E: mitral A/E' ratio. 


\section{DISCUSSION}

In this study examining the effects of levosimendan and dobutamine treatments administered to patients with decompensated heart failure on a variety of echocardiographic parameters, significant differences were noted in only a limited number of measurements. In both groups, LVEF and LVET significantly increased at the end of both levosimendan and dobutamine infusions with a similar extent. However, the results revealed that levosimendan significantly shortened QS2i while dobutamine had no effect. Furthermore, we postulated that levosimendan have a fairly stronger positive inotropic effect than dobutamine via shortening QS2i.

Until now, several studies have been performed to compare the clinical benefits of levosimendan and dobutamine in the treatment of heart failure. Among these, the LIDO study (Levosimendan Infusion versus Dobutamine in severe low Output heart failure) enrolling a total of 203 NYHA Class II-III patients reported a significantly higher number of patients with favorable hemodynamic response after 24 hours of treatment as well as a reduced 30-day mortality in the levosimendan group ${ }^{8}$. CASINO study (Calcium Sensitizer or Inotrope or None in Low-Output Heart Failure) was the first to compare levosimendan, dobutamine, and placebo, with levosimendan showing significant superiority over dobutamine in terms of mortality reduction ${ }^{9}$. In SURVIVE (Survival of Patients with Acute Heart Failure in Need of Intravenous Inotropic Support), the mortality benefit in favor of levosimendan observed in initial days disappeared at 180 days 10 .

The effect of these two agents on echocardiographic parameters has also been subject to some research. Duygu $\mathrm{H}$ et al. compared dobutamine and levosimendan with respect to $\mathrm{E} / \mathrm{E}$ ' ratio after 24-hour infusion and found a statistically significant reduction in levosimendan group, with no significant differences versus pre-treatment values in dobutamine patients ${ }^{11}$. In our study, we failed to detect significant differences both compared to baseline and between the study groups with regard to this parameter.

Duman D et al. compared these two agents in terms of their effects on diastolic function, and found no significant differences with pre-treatment measurements in dobutamine group, while a significant increase in mitral A, DT, and IVRT and a significant decrease in E/A ratio were reported for levosimendan ${ }^{12}$. On the other hand, no significant differences in diastolic parameters were found between the two agents in the current study.

In a study involving 40 patients with decompensated HF, Duygu $\mathrm{H}$ et al. observed significant increases in LVEF, Sm, Dt, and Em and significant decrease in E/A ratio and systolic pulmonary artery pressure (SPAP) among subjects receiving levosimendan as compared to those receiving dobutamine. In dobutamine patients, no significant alterations were found in systolic and diastolic LV parameters as well as in SPAP ${ }^{13}$. In our study, lateral and septal Sm velocity showed a significant increase following both inotropic agents, with no significant between-group differences. The $\mathrm{S}$ time exhibited a non-significant average decline of $3 \mathrm{msec}$ in dobutamine group, while a significant shortening of 10-12 msec was found in levosimendan group; however, the difference between the two groups was not statistically significant.

Poder P et al. found a significant reduction in QS2 among a group of patients with decompensated heart failure who received intravenous and oral levosimendan in the context of a Phase II study ${ }^{14}$. Also, another report suggested a shortening of QS2 by levosimendan 15. Consistent with these observations, we also found evidence of shortened QS2 during levosimendan treatment as compared to dobutamine. 
Positive inotropic agents generally only have a subtle effect on biochemical parameters, with many studies reporting no significant changes after treatment ${ }^{16}$. On the other hand, one study found a higher occurrence of hypokalemia among levosimendan recipients as compared to dobutamine ${ }^{10}$. Similarly, a significant decline in potassium levels was observed at the end of the treatment period in our study. This was the only significant treatment-related electrolyte alteration in our patients.

Duman D et al. compared the effects of dobutamine and levosimendan on SBP, DBP, and HR and found no significant differences 12, while Duygu $\mathrm{H}$ et al. observed a significant increase in these three parameters in dobutamine patients, with no significant changes with levosimendan ${ }^{13}$. In our study, dobutamine treated patients had an average increase of $11 \mathrm{mmHg}$ in SBP as compared to a $16.7 \mathrm{mmHg}$ reduction in levosimendan treated patients, suggesting a predilection toward hypertension and hypotension for these two treatments, respectively. Similarly, DBP increased significantly in dobutamine treated patients, while it decreased significantly in levosimendan treated patients, with a statistically significant difference between the two agents. Furthermore, dobutamine was associated with an increased heart rate.

According to our results, positive inotropic agents examined in our study had no unfavorable effects on biochemical and hematological parameters in patients with acute decompensated heart failure.

\section{Conclusion}

Our findings suggest that levosimendan and dopamine treatments are associated with differences only in a limited number of echocardiographic parameters. This study show that levosimendan and dobutamine effective in increasing LVET and in shortening PEP. However, levosimendan appears to have additional advantage over dobutamine in shortening QS2i, indicating a fairly strong positive inotropic effect. Further studies with larger sample size are warranted to better elucidate the effects of such treatments.

\section{References}

[1] Cleland JG, Khand A, Clark A. The heart failure epidemic: exactly how big is it? Eur Heart J. 2001;22(8):623-6.

[2] Jaagosild P, Dawson NV, Thomas C, Wenger NS, Tsevat J, Knaus WA, et al. Outcomes of acute exacerbation of severe congestive heart failure: quality of life, resource use, and survival. SUPPORT Investigators. The Study to Understand Prognosis and Preferences for Outcomes and Risks of Treatments. Arch Intern Med. 1998;158(10):1081-9.

[3] Cowie MR, Mosterd A, Wood DA, Deckers JW, Poole-Wilson PA, Sutton GC, et al. The epidemiology of heart failure. Eur Heart J. 1997;18(2):208-25.

[4] Packer M, Carver JR, Rodeheffer RJ, Ivanhoe RJ, DiBianco R, Zeldis SM, et al. Effect of oral milrinone on mortality in severe chronic heart failure. The PROMISE Study Research Group. N Engl J Med. 1991;325(21):1468-75.

[5] Slawsky MT, Colucci WS, Gottlieb SS, Greenberg BH, Haeusslein E, Hare J, et al. Acute hemodynamic and clinical effects of levosimendan in patients with severe heart failure. Study Investigators. Circulation. 2000;102(18):2222-7.

[6] Singh BN, Lilleberg J, Sandell EP, Ylönen V, Lehtonen L, Toivonen L. Effects of levosimendan on cardiac arrhythmia: electrophysiologic and ambulatory electrocardiographic findings in phase II and phase III clinical studies in cardiac failure. Am J Cardiol. 1999;83(12 (Suppl. 2)):16-20. 
[7] Dernellis J, Panaretou M. Effects of levosimendan on restrictive left ventricular filling in severe heart failure: a combined hemodynamic and Doppler echocardiographic study. Chest. 2005;128(4):2633-9.

[8] Follath F, Cleland JG, Just H, Papp JG, Scholz H, Peuhkurinen K, et al. Efficacy and safety of intravenous levosimendan compared with dobutamine in severe low-output heart failure (the LIDO study): a randomised double-blind trial. Lancet. 2002;360(9328):196-202.

[9] Zairis MN, Apostolatos C, Anastasiadis P, Mytas D, Katsaris C, Kouris N, et al. The effect of a calcium sensitizer or an inotrope or none in chronic low output decompensated heart failure: results from the calcium sensitizer or inotrope or none in low output heart failure study (CASINO). J Am Coll Cardiol. 2004;43((Suppl 1)):206-7.

[10] Mebazaa A, Nieminen MS, Packer M, Cohen-Solal A, Kleber FX, Pocock SJ, et al. Levosimendan vs dobutamine for patients with acute decompensated heart failure: the SURVIVE Randomized Trial. JAMA. 2007;297(17):1883-91.

[11] Duygu H, Ozerkan F, Nalbantgil S, Zoghi M, Akilli A, Akin M, et al. Effect of levosimendan on E/E' ratio in patients with ischemic heart failure. Int J Cardiol. 2008;123(2):201-3.

[12] Duman D, Palit F, Simsek E, Bilgehan K, Sacide A. Effects of levosimendan versus dobutamine on left atrial function in decompensated heart failure. Can J Cardiol. 2009;25(10):e353-6.

[13] Duygu H, Turk U, Ozdogan O, Akyuz S, Kirilmaz B, Alioglu E, et al. Levosimendan versus dobutamine in heart failure patients treated chronically with carvedilol. Cardiovasc Ther. 2008;26(3):182-8.

[14] Poder P, Eha J, Sundberg S, Antila S, Heinpalu M, Loogna I, et al. Pharmacokineticpharmacodynamic interrelationships of intravenous and oral levosimendan in patients with severe congestive heart failure. Int J Clin Pharmacol Ther. 2003;41(8):365-73.

[15] Lehtonen L, Sundberg S. The contractility enhancing effect of the calcium sensitiser levosimendan is not attenuated by carvedilol in healthy subjects. Eur J Clin Pharmacol. 2002;58(7):449-52.

[16] Maeda K, Tsutamoto T, Wada A, Mabuchi N, Hayashi M, Tsutsui T, et al. High levels of plasma brain natriuretic peptide and interleukin-6 after optimized treatment for heart failure are independent risk factors for morbidity and mortality in patients with congestive heart failure. $\mathbf{J}$ Am Coll Cardiol. 2000;36(5):1587-93. 